\title{
Spontaneous emission factor in oxide confined vertical-cavity lasers
}

\author{
D. V. Kuksenkov a) and H. Temkin \\ Department of Electrical Engineering, Texas Tech University, Lubbock, Texas 79409
}

K. L. Lear and H. Q. Hou

Sandia National Laboratories, Albuquerque, New Mexico 87185

(Received 3 September 1996; accepted for publication 5 November 1996)

\begin{abstract}
We report on measurements of the spontaneous emission factor for oxide-confined InGaAs vertical cavity surface emitting lasers. The spontaneous emission factor is determined as a function of the active layer volume from the measurement of small-signal harmonic distortion at threshold. For a $3 \times 3 \mu \mathrm{m}$ oxide aperture device we obtain spontaneous emission factor of $4.2 \cdot 10^{-2}$ at room temperature. (c) 1997 American Institute of Physics. [S0003-6951(97)03501-8]
\end{abstract}

The possibility of controlling spontaneous emission in semiconductor microcavities has attracted considerable attention due to predictions of essentially thresholdless lasing and enhanced modulation bandwidth. ${ }^{1}$ The spontaneous emission factor $\beta$ has been measured for buried mesa VCSELs with dielectric mirrors, ${ }^{2}$ gain-guided VCSELs, ${ }^{3,4}$ optically pumped lasers with planar resonators, ${ }^{5}$ and for air-post mesa VCSELs at low temperatures. ${ }^{6}$ The $\beta$ factor of oxideconfined VCSELs was estimated from the spectral and angular width of the spontaneous emission. ${ }^{7}$ The results reported thus far differ significantly from structure to structure and the measurement conditions, with the highest values of $\beta$ $\sim 10^{-2}$ observed at low temperatures. ${ }^{5,6}$

The enhancement in the value of $\beta$ is expected to significantly affect the laser performance when the lateral dimensions of the cavity are reduced to less than $1 \mu \mathrm{m} .{ }^{8,9}$ Unfortunately, VCSEL size scaling is limited by increasing optical loss and consequently the threshold carrier density. In addition, the enhancement of spontaneous emission in a microcavity formed by distributed Bragg reflectors is limited by the finite width of the reflection band and the optical field penetration into the mirrors. ${ }^{10}$

Oxide confined VCSELs are characterized by significantly reduced internal loss and have already demonstrated record performance levels in terms of the threshold current and power conversion efficiency. ${ }^{11}$ Lasers with the lateral cavity dimensions below $\sim 1 \mu \mathrm{m}$ appear feasible.

Devices used in our measurements are based on three InGaAs quantum wells in the active region, and are designed to emit at $980 \mathrm{~nm}$. Two quarter-wavelength $\mathrm{Ga}_{0.02} \mathrm{Al}_{0.98} \mathrm{As}$ layers, one above and one below the active region, are partially oxidized to form the current aperture. Devices with the aperture sizes in the range of 3-25 $\mu \mathrm{m}$ exhibit threshold currents from 0.27 to $3.3 \mathrm{~mA}$, and threshold voltages of 2.6$1.4 \mathrm{~V}$.

The conventional method of estimating the $\beta$ factor is based on the rate equations fitting the measured light-current $(L-I)$ curve. $^{12}$ When applied to VCSELs, this technique is likely to be inaccurate. In very small devices, the temperature of the active region, and therefore the threshold and efficiency, are dependent on the drive current as a result of self-heating, and these effects require careful compensation. Larger index guided devices lase in multiple transverse

${ }^{a)}$ Electronic mail: DKuksenkov@coe2.coe.ttu.edu modes [see Fig. 1(b)], and the modal distribution is also dependent on the drive current, even though calculations usually assume it to be constant.

An alternative technique is used here to determine $\beta$. It is based on the measurement of small-signal harmonic distortion at threshold, first suggested by Goodwin and Garside $^{13}$ and recently applied to VCSELs. ${ }^{5}$ This method can be very accurate since all the measurements are made at or near threshold, and therefore all the current induced changes are insignificant. To make the measurement even more precise we modify the previously derived expressions for $\beta^{13}$ to take into account the nonzero transparency current.

We restrict our analysis to the case of a single transverse mode. As illustrated in Fig. 1, the number of transverse modes in the VCSEL spectrum at threshold can be quite large, but the zeroth-order mode always dominates. Singlemode rate equations are written as:

$$
\begin{aligned}
& \frac{d N}{d t}=\frac{\eta_{i} I}{e V_{a}}-\frac{N}{\tau_{n}}-A\left(N-N_{0}\right) S, \\
& \frac{d S}{d t}=\Gamma A\left(N-N_{0}\right) S-\frac{S}{\tau_{p}}+\Gamma \beta \frac{N}{\tau_{r}},
\end{aligned}
$$
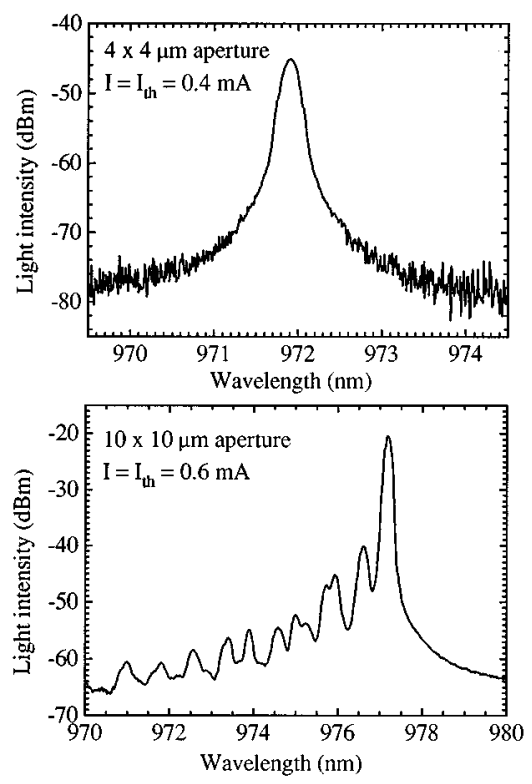

FIG. 1. Near-threshold spectra of $4 \times 4$ and $10 \times 10 \mu \mathrm{m}$ oxide aperture devices. 
where $N(S)$ are the carrier (photon) densities, $\eta_{i}$ is the injection efficiency, $I$ is the drive current, $e$ is the elementary charge, $V_{a}$ is the active region volume, $A$ is the differential gain, $\Gamma$ is the optical confinement factor, $N_{0}$ is the carrier density at transparency, $\tau_{p}$ is the photon lifetime in the cavity, $\tau_{n}$ is the carrier lifetime, and $\tau_{r}$ is the radiative carrier lifetime.

In general, $\tau_{n}<\tau_{r}$, and we use the following definition of the radiative efficiency $\eta_{r}$ :

$$
\eta_{r}=\frac{\tau_{n}}{\tau_{r}}=\frac{\text { radiative recombination rate }}{\text { total recombination rate }} .
$$

After that, our analysis closely follows the guidelines of Ref. 13. We assume small-signal modulation in the form of $x$ $=x_{0}+\gamma \sin (\omega t)$, where $x$ is the normalized pump variable and $\gamma$ is the modulation index

$$
x=\frac{I-I_{\mathrm{tr}}}{I_{\mathrm{th}}-I_{\mathrm{tr}}} ; \quad \gamma=\frac{I_{m}}{I_{\mathrm{th}}-I_{\mathrm{tr}}},
$$

where $I_{\text {th }}$ is the threshold current, $I_{\text {tr }}$ is the transparency current, and $I_{m}$ is the modulation current amplitude. From harmonic analysis of the steady-state solution of (1), we find that the second harmonic peaks very close to threshold at $x_{\text {peak }}=1-2 \beta \eta_{r}$, and the ratio of the amplitudes of the second and fundamental harmonics at $x_{\text {peak }}$ is given by

$$
|R|=\frac{1}{4} \frac{\gamma}{\sqrt{4 \beta \eta_{r}\left(1+x_{t r}\right)}},
$$

where $x_{\mathrm{tr}}=I_{\mathrm{tr}} /\left(I_{\mathrm{th}}-I_{\mathrm{tr}}\right)$ is the normalized transparency current. Finally, we obtain

$$
\beta=\frac{1}{4 \eta_{r}\left(1+x_{\mathrm{tr}}\right)}\left(\frac{\gamma}{4 R}\right)^{2} .
$$

It is interesting to compare (5) to the $\beta^{\prime}=\left(\gamma^{\prime} / 4 R\right)^{2}$, obtained in Ref. 13 [note that $\gamma^{\prime}=I_{m} / I_{\text {th }}$ is different from $\gamma$ in our Eq. (5)]. For $\eta_{r}=1$ and the transparency current equal to one third of the threshold current, we have $\beta \approx 1.5 \beta^{\prime}$. When the approach of Ref. 13 is used without a correction for the nonzero transparency current, ${ }^{5}$ the resulting values of the $\beta$ factor are underestimated by at least $50 \%$.

Small-signal sinusoidal modulation used in our experiments is produced by a low-distortion synthesized rf generator. The modulation signal at $30 \mathrm{kHz}$ is mixed with the dc bias using a standard bias- $T$. To ensure the validity of the small-signal approximation the modulation index is kept below $\gamma=2 \%$. The laser is mounted on a heat-sink and temperature stabilized at $T=20{ }^{\circ} \mathrm{C}$. A cleaved end of standard multimode (50 $\mu \mathrm{m}$ core) optical fiber, positioned several millimeters away from the laser surface, is used to collect the light and to spatially filter the spontaneous emission. The light is then put through an optical spectrum analyzer acting as a filter with the resolution bandwidth set at $0.2 \mathrm{~nm}$, selecting only the zeroth-order transverse mode of the laser. The spatially and spectrally filtered emission is detected by an InGaAs $p-i-n$ photodiode, and the amplitudes of the fundamental and second harmonics are measured by a lock-in amplifier.

The measured harmonic amplitudes are plotted in Fig. 2 as a function of drive current for two lasers, $5 \times 5$ and $10 \times 10$

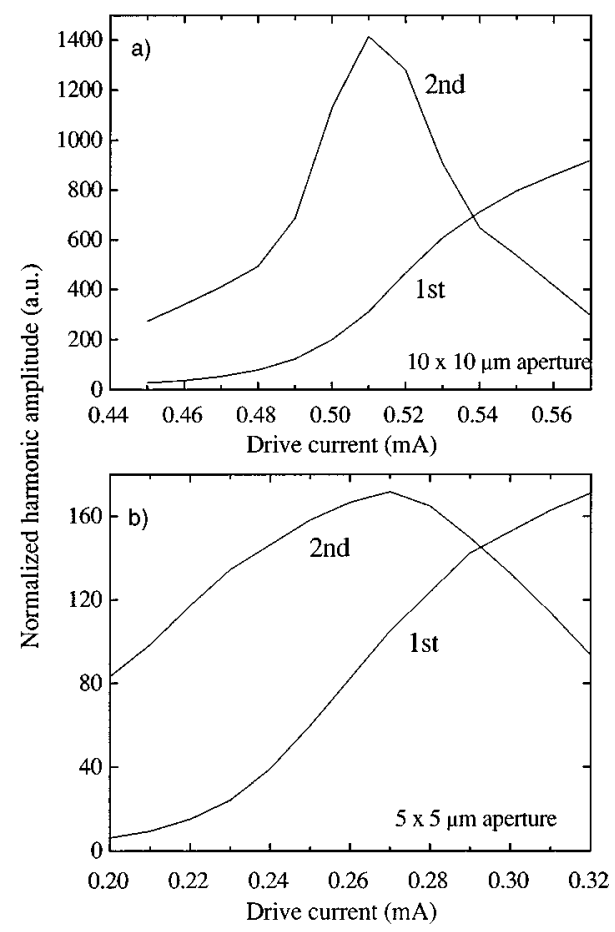

FIG. 2. Harmonic amplitude dependence on the normalized drive current for two VCSELs of different sizes. The amplitude of the fundamental harmonic is normalized by $\gamma$ and the amplitude of the second harmonic is normalized by $\gamma^{2} / 4$.

$\mu \mathrm{m}$ in size. It is clear from Fig. 2 that the measured harmonics behave as expected from theoretical analysis.

The knowledge of three additional parameters, $I_{\mathrm{th}}, I_{\mathrm{tr}}$, and $\eta_{r}$, is needed in order to extract the $\beta$ factor. The threshold currents were determined from the kink position in the measured differential current-voltage $(I-V)$ characteristics $I d V / d I(I)$. The transparency current $I_{\mathrm{tr}}$ is determined from the measurement of photoinduced current dependence on dc bias, using the device under test as a photodetector and a second VCSEL, of the same kind, as a source of light. ${ }^{14}$ The value of radiative efficiency $\eta_{r}$ can be obtained from a direct measurement of the differential carrier lifetime dependence on the drive current below threshold, ${ }^{15}$ or estimated from comparison of the actual injection current density at threshold with the one predicted for an ideal laser with $\eta_{r}=1$, as done in Ref. 6. We assume $\eta_{r}=1$ for all devices meaning that it is the product of $\eta_{r} \beta$, rather than the $\beta$ factor itself being reported on.

The measured values of the spontaneous emission factor are plotted in Fig. 3 as a function of the cavity width. It is clear that $\beta$ scales as the inverse of the active layer volume. This type of scaling is predicted by the classical electromagnetic theory and is attributed to a decrease in the number of available cavity modes. A rigorous calculation of spontaneous emission coupling into the lasing mode of a distributed Bragg reflector surface emitting microcavity laser with quantum well (QW) active region ${ }^{16}$ shows that for cavity widths $a \gg \lambda / n_{\text {eff }}$, where $\lambda$ is the resonant wavelength and $n_{\text {eff }}$ is the effective refractive index, one can approximate:

$$
\beta_{\mathrm{QW}} \approx \xi m \beta_{\text {bulk }} .
$$

The coefficient $m$ accounts for the dipole radiation enhance- 


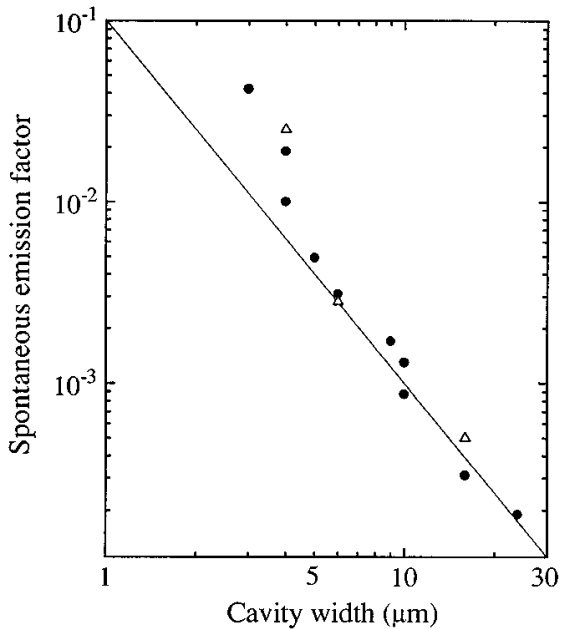

FIG. 3. Spontaneous emission factor dependence on the cavity width: solid circles-experimental data obtained from harmonic distortion measurements, open triangles-experimental data obtained from the fit to the measured $L-I$ curve, solid line-theoretical result.

ment in the direction perpendicular to the plane of the QW and is equal to 1.5. The coefficient $\xi$ accounts for the change in modal density distribution in QW and varies from 2 to 0 depending on the relative position of the QW and the mode field. The lasers used here are designed with the QWs at the center of the $\lambda$ cavity and we calculate $\xi \approx 1.8$. The $\beta_{\text {bulk }}$ is calculated from classical electromagnetic theory as one half of the inverse value of the total number of modes in the emission spectrum: ${ }^{17}$

$$
\beta_{\text {bulk }}=\epsilon \frac{\lambda^{4}}{4 \pi n_{\mathrm{eff}}^{3} V \Delta \lambda},
$$

where $\epsilon$ is the power confinement factor of a mode, $V$ is the waveguide volume, and $\Delta \lambda$ is the full width half maximum (FWHM) of the spontaneous emission spectrum.

The calculated $\beta_{\mathrm{QW}}$ is plotted in Fig. 3 (solid line) as a function of the cavity width. Since for the smallest of our devices $(3 \times 3 \mu \mathrm{m})$ the cavity width $a \approx 11 \lambda / n_{\text {eff }}$, we expect our experimental data to follow the straight line. Indeed, for $a>5 \mu \mathrm{m}$, the agreement is evident. However, for smaller sizes, the measured $\beta$ is almost a factor of 4 higher than that predicted by theory. This disagreement needs to be explained. For the smallest lasers it is difficult to assess the cavity size and waveguide parameters with sufficient accuracy. In the calculation we assumed the cavity size to be equal to that of the oxide aperture. We also assumed a steplike index variation due to the confining oxide. The actual index profile can be affected by the edge diffraction and self-heating, especially in small devices. A $\sim 1 \mu \mathrm{m}$ overestimate in the cavity size would place the small device data points back on the calculated line.

We also observe a clear dependence of the spontaneous emission factor on the threshold carrier density. For the two devices of the same size the lower threshold laser always exhibits higher $\beta$ (see Fig. 3). Since the measured transparency current is almost constant for a given device size, the difference in threshold is assumed to be mostly due to the difference in optical loss. The decrease in $\beta$ with increasing carrier density can then be attributed to the broadening of the spontaneous emission spectrum as well as increased optical loss.

To compare the two methods of determining the spontaneous emission factor, we fit rate equations to measured $L-I$ characteristics for selected devices. This measurement is done for the zeroth-order transverse mode in the vicinity of threshold. Data points obtained in this way are plotted as triangles in Fig. 3. For mid-size devices $(5-10 \mu \mathrm{m}$ cavity width) the best fit to the $L-I$ curve gives approximately the same $\beta$ value as those obtained by harmonic distortion analysis.

In conclusion, we report the results of spontaneous emission factor measurement for oxide-confined InGaAs vertical cavity lasers emitting at $980 \mathrm{~nm}$. The $\beta$ factor is determined from the measurement of the small-signal harmonic distortion at threshold. For a $3 \times 3$ oxide aperture device we obtain $\beta=4.2 \cdot 10^{-2}$ which is, to our knowledge, the highest value reported for any VCSELs to date.

The authors thank J. Banas for technical assistance. The work at Sandia was supported in part by the United States Department of Energy under Contract DE-AC0494AL85000, work at Texas Tech is supported by the National Science Foundation and the Maddox Foundation.

${ }^{1}$ Y. Yamamoto, S. Machida, and G. Bjork, Phys. Rev. A 44, 657 (1991).

${ }^{2}$ F. Koyama, K. Morito, and K. Iga, IEEE J. Quantum Electron. 27, 1410 (1991)

${ }^{3}$ G. Shtengel, H. Temkin, T. Uchida, M. Kim, P. Brusenbach, and C. Parsons, Appl. Phys. Lett. 64, 1062 (1994).

${ }^{4}$ G. Bjork, H. Heitmann, and Y. Yamamoto, Phys. Rev. A 47, 4451 (1993).

${ }^{5}$ Y.-G. Zhao, J. G. McInerney, and R. A. Morgan, IEEE Photonics Technol. Lett. 7, 1231 (1995).

${ }^{6}$ R. J. Ram, E. Goobar, M. G. Peters, L. A. Coldren, and J. E. Bowers, IEEE Photonics Technol. Lett. 8, 599 (1996).

${ }^{7}$ D. G. Deppe, D. L. Huffaker, J. Shin, and Q. Deng, IEEE Photonics Technol. Lett. 7, 965 (1995).

${ }^{8}$ G. Bjork and Y. Yamamoto, IEEE J. Quantum Electron. 27, 2386 (1991).

${ }^{9}$ T. Baba, T. Hamano, F. Koyama, and K. Iga, IEEE J. Quantum Electron. 27, 1347 (1991)

${ }^{10}$ R. J. Ram, D. I. Babic, R. A. York, and J. E. Bowers, IEEE J. Quantum Electron. 31, 399 (1995).

${ }^{11}$ K. D. Choquette, K. L. Lear, R. P. Schneider, Jr., K. M. Geib, J. J. Figiel, and R. Hull, IEEE Photonics Technol. Lett. 7, 1237 (1995).

${ }^{12}$ Y. Suematsu, S. Akiba, and T. Hong, IEEE J. Quantum Electron. QE13, 596 (1995).

${ }^{13}$ J. C. Goodwin and B. K. Garside, IEEE J. Quantum Electron. QE18, 1264 (1982).

${ }^{14}$ D. V. Kuksenkov, H. Temkin, and S. Swirhun, Appl. Phys. Lett. 66, 1720 (1995).

${ }^{15}$ J. W. Bae, G. Shtengel, D. Kuksenkov, H. Temkin, and P. Brusenbach, Appl. Phys. Lett. 66, 2031 (1995).

${ }^{16}$ T. Baba, T. Hamano, F. Koyama, and K. Iga, IEEE J. Quantum Electron. 28, 1310 (1991).

${ }^{17}$ Y. Suematsu and K. Furuya, Trans. IECE Jpn. E-60, 467 (1977). 